\title{
Bernard Kalaora, 2010, Rivages en devenir. Des horizons pour le Conservatoire du littoral, Paris, La Documentation française, 280 pages.
}

\section{Clarisse Cazals}

\section{(2) OpenEdition}

1 Journals

\section{Édition électronique}

URL : http://journals.openedition.org/developpementdurable/10190

DOI : 10.4000/developpementdurable.10190

ISSN : 1772-9971

Éditeur

Association DD\&T

\section{Référence électronique}

Clarisse Cazals, «Bernard Kalaora, 2010, Rivages en devenir. Des horizons pour le Conservatoire du littoral, Paris, La Documentation française, 280 pages. », Développement durable et territoires [En ligne], Vol. 5, n¹ | Février 2014, mis en ligne le 04 février 2014, consulté le 22 septembre 2020. URL : http:// journals.openedition.org/developpementdurable/10190; DOI : https://doi.org/10.4000/

developpementdurable.10190

Ce document a été généré automatiquement le 22 septembre 2020

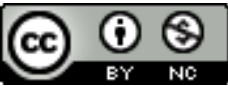

Développement Durable et Territoires est mis à disposition selon les termes de la licence Creative Commons Attribution - Pas d'Utilisation Commerciale 4.0 International. 


\section{Bernard Kalaora, 2010, Rivages en devenir. Des horizons pour le Conservatoire du littoral, Paris, La Documentation française, 280 pages.}

\section{Clarisse Cazals}

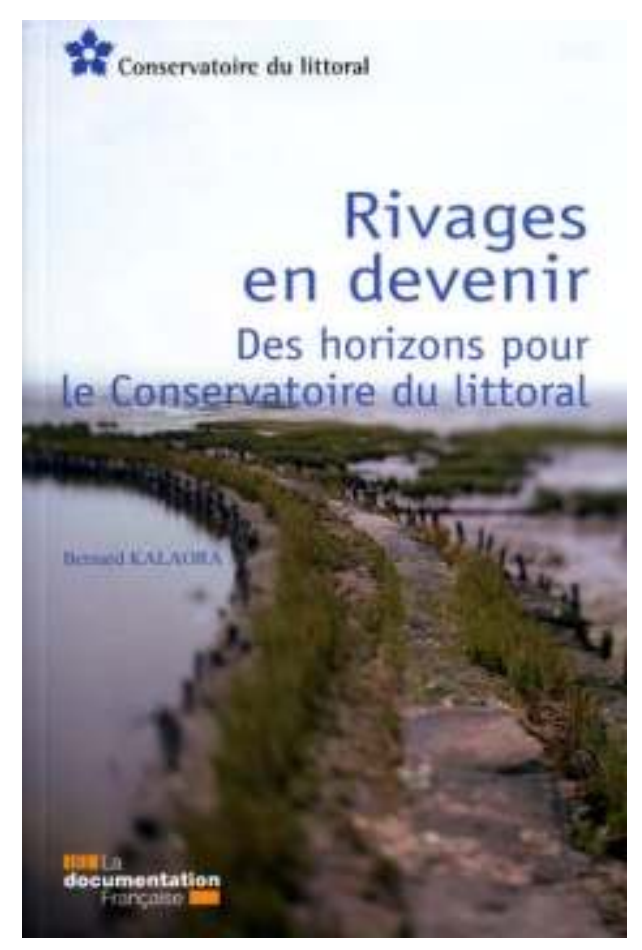

Il n'est plus nécessaire de présenter B. Kalaora dans le champ des spécialistes de l'environnement. Pionnier au sein des sciences humaines et sociales, et plus précisément en sociologie, pour le développement des recherches sur les questions environnementales, il est également impliqué depuis longtemps dans l'action publique 
environnementale. Fidèle à ce positionnement original entre la recherche et l'engagement politique, dans l'ouvrage «Rivages en devenir. Des horizons pour le Conservatoire du littoral », B. Kalaora, membre du conseil scientifique du Conservatoire $\mathrm{du}$ Littoral, présente une analyse des mutations de la trajectoire patrimoniale empruntées par le Conservatoire du littoral. Ce travail est imprégné de l'expérience des «Ateliers» du Conservatoire qu'il a animés depuis 1993. Dans un ouvrage découpé en cinq parties, l'auteur analyse les mutations des logiques d'action du Conservatoire du Littoral depuis sa création et montre ainsi l'évolution d'une politique patrimoniale s'appuyant uniquement sur la propriété foncière vers l'intégration de modes de gouvernance participatifs.

2 La première partie du livre permet d'apprécier les multiples dimensions patrimoniales qui ont inspiré et façonné l'action du Conservatoire. La protection du littoral par la propriété foncière s'appuie sur une production artistique très riche, qu'elle soit littéraire, photographique ou picturale. Ainsi, face aux nombreuses représentations du littoral issues de l'expérience et à l'émergence tardive de référentiels scientifiques, les paysages et les espaces remarquables qui s'inscrivent bien au croisement de la culture et de la nature seront au cœur des préoccupations du Conservatoire. Le littoral ne laisse personne indifférent. Toutefois, dans la lignée de l'analyse réalisée par Olivier Godard (Godard 2004) au sujet du développement durable, la mobilisation du modèle des cités (Boltanski and Thévenot 1991) permet de distinguer différents arguments justifiant la protection du littoral. Le Conservatoire a pour mission de les rendre compatibles et légitimes de façon à permettre la reconnaissance d'un patrimoine littoral par la protection de sites emblématiques.

La deuxième partie de l'ouvrage est consacrée aux objets qui incarnent ce patrimoine littoral. La maîtrise foncière étant au fondement de l'action du Conservatoire, le patrimoine littoral est avant tout constitué d'espaces quasi unanimement qualifiés de remarquables. Les îles et les jardins y tiennent une place privilégiée. Cependant, les acteurs directement ou indirectement concernés par ces lieux ne partagent pas naturellement tous les mêmes visions des usages compatibles avec la mise en place d'un dispositif de protection caractéristique du processus de patrimonialisation. A ce titre, l'usage agricole a longtemps été considéré étranger à ce processus. Néanmoins, les modes de production agricole extensifs sont peu à peu reconnus pour la place qu'ils tiennent dans la conservation des paysages, des bâtis et des espèces et qui sont des composantes du patrimoine littoral. Les propriétés du Conservatoire sont également des lieux privilégiés pour la mise en œuvre de politiques patrimoniales fondées sur des réglementations environnementales internationales, des politiques d'inventaires et de recensement des espèces. Si les artistes, les experts paysagistes et les scientifiques ont tenu un rôle central dans le développement de telles politiques sur le littoral, celui-ci tend à évoluer fortement, voire à être remis en cause, avec l'émergence de modes de gouvernance plus flexibles et décentralisés pour ces espaces protégés.

4 La troisième partie analyse les mutations de l'action publique sur le littoral. La politique patrimoniale du Conservatoire reposant sur la conservation de sites naturels remarquables est bousculée par l'importance de la crise environnementale et la nécessité d'envisager la protection dans une perspective dynamique de gestion intégrée. La propriété foncière tout en restant au cœur de l'action du Conservatoire se doit de prendre en compte un plus grand nombre d'acteurs, d'activités économiques ainsi que leurs interactions et d'appréhender les sites du Conservatoire en réseau. 
5 La quatrième partie présente les différentes facettes qu'implique le changement d'échelle de l'action du Conservatoire. Les enjeux environnementaux globaux comme la préservation de la biodiversité, le changement climatique et les risques d'érosion et de submersion sont désormais au cœur des préoccupations du Conservatoire. Dès lors, les décisions en termes de protection ne peuvent être indépendantes de celles des acteurs locaux, nationaux et internationaux et échapper au contexte d'incertitude radicale qui les caractérise. Elles visent à dépasser la protection d'un patrimoine naturel et culturel répondant à une logique d'inventaire pour promouvoir celle reposant sur la gestion des espaces littoraux. Dans ces conditions, tout comme les gestionnaires des espaces littoraux, les agriculteurs et les touristes deviennent des partenaires incontournables pour gérer ces espaces remarquables. Le maintien d'activités productives respectueuses de l'environnement et l'ouverture au public réglementée sont désormais perçus comme des atouts pour la protection de ces sites.

6 Finalement, la cinquième partie entérine les mutations de l'univers du littoral auxquelles le Conservatoire a dû s'adapter. Dans un contexte où la prégnance du développement durable s'accentue par l'intermédiaire des institutions internationales, la vision traditionnelle du patrimoine naturel s'appuyant sur une politique d'espaces protégés est un axe central de la mise en œuvre du développement durable à l'échelle locale qui est contraint d'évoluer. Il doit s'ouvrir à la prise en compte des objectifs économiques et sociaux, d'autant que la nature est désormais considérée comme un espace appropriable par divers acteurs. Dès lors, le Conservatoire bénéficie de son expérience, diverse et originale, dans les relations aux gestionnaires de sites pour évoluer vers un rôle de propriétaire-partenaire, et ainsi être au cœur de la politique locale du développement durable.

7 En conclusion, l'ouvrage met bien en exergue une certaine remise en cause de la politique du patrimoine naturel à l'origine de l'action du Conservatoire du littoral, qui laisse la place à de nouvelles formes d'action publique concomitantes à la mise en œuvre d'une gouvernance adaptée aux enjeux du développement durable. Les difficultés d'adaptation des agents du Conservatoire à ce contexte marqué par de nombreuses controverses scientifiques et sociales ont été également abordées. Ainsi, le Conservatoire est un acteur majeur du développement harmonieux des usages, marchands ou non marchands, compatibles avec la préservation des espaces littoraux. Il est donc au cœur de la gestion des tensions entre préservation et usage qui, selon le renouvellement de l'analyse économique du patrimoine (Barrère, Barthelemy et al. 2005), participe à l'émergence et à la reconnaissance de diverses formes de patrimoine et implique de l'inscrire dans une perspective dynamique.

Comme un grand nombre d'articles consacrés à l'analyse du processus de gestion intégrée des zones côtières (GIZC) qui est la référence de toutes politiques littorales, cet ouvrage retrace la lente progression vers la mise en place d'une gestion innovante supposant l'intégration d'éléments multiples mais qui reste marquée par de nombreux obstacles comme par des mutations irréversibles. Alors que l'articulation de la gouvernance territoriale avec la gestion des activités maritimes est désormais au cœur de ce processus, l'analyse proposée par l'auteur nourrit les nombreuses interrogations suscitées par les nouveaux enjeux de gestion de cette économie bleue. 


\section{BIBLIOGRAPHIE}

Barrère C., Barthelemy D. et al., 2005, Réinventer le Patrimoine : de la culture à l'économie, une nouvelle pensée du patrimoine ?, Paris, Édition l'Harmattan.

Boltanski L. et. Thévenot L, 1991, De la justification : les Économies de la Grandeur, Paris, Gallimard, coll. NRF Essais.

Godard O., 2004, « De la pluralité des ordres - Les problèmes d'environnement et de développement durable à la lumière de la théorie de la justification », Géographie, Économie, Société $n^{\circ}$ 6, p. 303-330.

\section{AUTEUR}

\section{CLARISSE CAZALS}

Clarisse Cazals est économiste, chargée de recherche à Istrea. Ses travaux portent principalement sur les dimensions patrimoniales des activités économiques. Elle s'intéresse à la diversité des logiques d'acteurs participant à la variété des formes de patrimoine, notamment aux divergences d'intérêt dont elles sont porteuses mais aussi aux dispositifs de coordination susceptibles de la rendre compatibles. 